\title{
RESEARCH
}

Open Access

\section{Genotype-phenotype correlation and description of two novel mutations in Iranian patients with glycogen storage disease $1 b$ (GSD1b)}

Maryam Eghbali ${ }^{1}$, Maryam Abiri ${ }^{2}$, Saeed Talebi ${ }^{2}$, Zahra Noroozi ${ }^{3}$, Marjan Shakiba ${ }^{4}$, Parastoo Rostami ${ }^{5}$, Hosein Alimadadi ${ }^{6}$, Mehri Najafi ${ }^{6}$, Fatemeh Yazarlou ${ }^{1}$, Ali Rabbani ${ }^{5}$ and Mohammad Hossein Modarressi ${ }^{*}$

\begin{abstract}
Background: Glycogen storage disease (GSD) is a rare inborn error of the synthesis or degradation of glycogen metabolism. GSD1, the most common type of GSD, is categorized into GSD1a and GSD1b which caused by the deficiency of glucose-6-phosphatase (G6PC) and glucose-6-phosphate transporter (SLC37A4), respectively. The high rates of consanguineous marriages in Iran provide a desirable context to facilitate finding the homozygous pathogenic mutations. This study designates to evaluate the clinical and genetic characteristics of patients with GSD1b to assess the possible genotype-phenotype correlation.

Results: Autozygosity mapping was performed on nineteen GSD suspected families to suggest the causative loci. The mapping was done using two panels of short tandem repeat (STR) markers linked to the corresponding genes. The patients with autozygous haplotype block for the markers flanking the genes were selected for direct sequencing. Six patients showed autozygosity in the candidate markers for SLC37A4. Three causative variants were detected. The recurrent mutation of c.1042_1043delCT (p.Leu348Valfs*53) and a novel missense mutation of c.365G > A (p.G122E) in the homozygous state were identified in the SLC37A4. In silico analysis was performed to predict the pathogenicity of the variants. A novel whole SLC37A4 gene deletion using long-range PCR and sequencing was confirmed as well. Severe and moderate neutropenia was observed in patients with frameshift and missense variants, respectively. The sibling with the whole gene deletion has shown both severe neutropenia and leukopenia.
\end{abstract}

Conclusions: The results showed that the hematological findings may have an appropriate correlation with the genotype findings. However, for a definite genotype-phenotype correlation, specifically for the clinical and biochemical phenotype, further studies with larger sample sizes are needed.

Keywords: GSD1b, Autozygosity mapping, Novel variants, Genotype-phenotype correlation

\footnotetext{
* Correspondence: modaresi@tums.ac.ir

${ }^{1}$ Department of Medical Genetics, Faculty of Medicine, Tehran University of

Medical Sciences, Tehran, Iran

Full list of author information is available at the end of the article
}

(c) The Author(s). 2020 Open Access This article is distributed under the terms of the Creative Commons Attribution 4.0 International License (http://creativecommons.org/licenses/by/4.0/), which permits unrestricted use, distribution, and reproduction in any medium, provided you give appropriate credit to the original author(s) and the source, provide a link to the Creative Commons license, and indicate if changes were made. The Creative Commons Public Domain Dedication waiver (http://creativecommons.org/publicdomain/zero/1.0/) applies to the data made available in this article, unless otherwise stated. 


\section{Background}

Glycogen storage diseases (GSDs) comprise a heterogeneous group of rare inborn errors of metabolism disorders caused by deficiency of the specific enzymes in glycogen degradation and synthesis. Depending on the impaired enzyme and affected organ, GSDs are classified into over 10 types. GSD1 is the most common liver impairment with an overall incidence of about 1:100,000 live births. It is categorized into 1a (GSD1a) and 1b (GSD1b). GSD1a is the more frequent type responsible for $>80 \%$ of GSD 1 patients [1] while GSD1b is estimated to represent $\sim 20 \%$ of cases [2].

Deficiency in glucose-6-phosphatase- $\alpha$ (G6Pase- $\alpha$ )/glucose-6-phosphate transporter (G6PT) complexes causes GSD1. This complex has a key role in maintaining glucose homeostasis through glycogenolysis and gluconeogenesis in liver, kidney, and intestine. G6Pase- $\alpha$ which is encoded by the G6PC gene catalyzes the hydrolysis of glucose-6-phosphate (G6P) to glucose and phosphate. The products will be transported into the lumen of the endoplasmic reticulum from the cytoplasm by G6PT (encoded by the SLC37A4 gene) [2, 3]. GSD1a disease is caused by the deficiency of the G6Pase- $\alpha$ which is located at the endoplasmic reticulum membrane. GSD1a and GSD1b patients represent similar metabolic phenotypes such as hypoglycemia, hepatomegaly, lactic acidemia, hyperlipidemia, and nephromegaly. Additionally, GSD 1b patients exhibit neutropenia and impaired neutrophil function; resulting in recurrent bacterial infections, inflammatory bowel disease (IBD), and aphthous stomatitis [4]. Nevertheless, not all GSD 1b patients manifest neutropenia, it may cause by either one or more modifiers on G6PT function or SLC37A4 mutations with residual transport activity [4, 5].

The diagnosis of GSD1 is based on the clinical symptoms, biochemical parameters, and G6Pase activity on liver biopsy tissues which is an invasive procedure. The clinical manifestations are not always useful to differentiate between GSD types $1 \mathrm{a}$ and $1 \mathrm{~b}$ patients [6]. The definitive diagnosis of the disease is established by molecular analysis of G6PC and SLC37A4.

The estimated rate of consanguineous marriages in Iran is about $38.6 \%$ [7] which provides an appropriate context for autozygosity mapping. This powerful tool helps us to quickly identify the possible defective gene flagged by autozygous blocks. Accordingly, in this project we identified candidate short tandem repeat (STR) markers flanking G6PC and SLC37A4 with acceptable heterozygosity in the selected population. Suitable families who showed autozygosity for the markers flanking SLC37A4 were selected for additional molecular genetics investigations. The aim of this study is to evaluate the clinical and genetic characteristics of patients in order to assess the possible genotype-phenotype correlation.

\section{Material and methods \\ Patients}

Patients were recruited from the Children Medical Center Hospital and the Mofid Children's Hospital in Tehran, Iran during January 2015-April 2019. Twenty Iranian patients from 19 unrelated families were investigated. Inclusion criteria were based upon clinical presentations of hepatomegaly, "doll face" and biochemical laboratory tests such as hypoglycemia, hypertriglyceridemia, hypercholesterolemia, hyperlactatemia, hyperuricemia, and elevated aspartate aminotransferase (AST) or alanine transaminase (ALT) suggestive of GSD.

The other laboratory measurement was cell blood count including neutrophil count and white blood cell count (WBC). In addition, patients were selected after confirmation by histological analysis of liver biopsy. All patients participating in this study had consanguineous parents. Genetic counseling was conducted and all subjects and/or their parents signed the consent form. The Ethical Committee of the Tehran University of Medical Sciences approved the study.

\section{Molecular genetics studies}

Human genomic DNA was isolated from peripheral leukocytes using Salting out method [8]. To indirectly find the possible mutated gene, autozygosity mapping was performed using the proper STR markers flanking SLC37A4 and G6PC genes. Six polymorphic STR markers linked to these genes were selected using Tandem Repeat Finder (TRF) and Sequence-based Estimation of Repeat Variability (SERV) software [9, 10]. An attempt was made to select markers with the length of 3-5 nucleotide repeats, high allelic heterogeneity and the nearest markers flanking upstream and downstream of the genes responsible for GSD1. The heterozygosity of the selected markers was assessed in 10 random and unrelated individuals. The selected markers were amplified with specific primers (the primer sequences are available upon request). Then the PCR products were analyzed by running on $10.0 \%$ polyacrylamide gels and silver nitrate staining. Then a haplotype map was drawn for each family (Fig. 1). Then exons and intron-exon boundaries of the candidate gene were sequenced (the primer sequences are available upon request) and were compared with the cDNA reference (NM_001164277 and NM_000151). PCR reaction for sequencing was performed in a total volume of $25 \mu$ l which included $8 \mu \mathrm{l}$ the Taq $2 \times$ Master Mix (Amplicon Company), $1 \mu \mathrm{l}$ of each $10 \mathrm{pM}$ primer, $13.5 \mu \mathrm{L} \mathrm{DH} 2 \mathrm{O}$ and $1.5 \mu \mathrm{l}$ of 50 


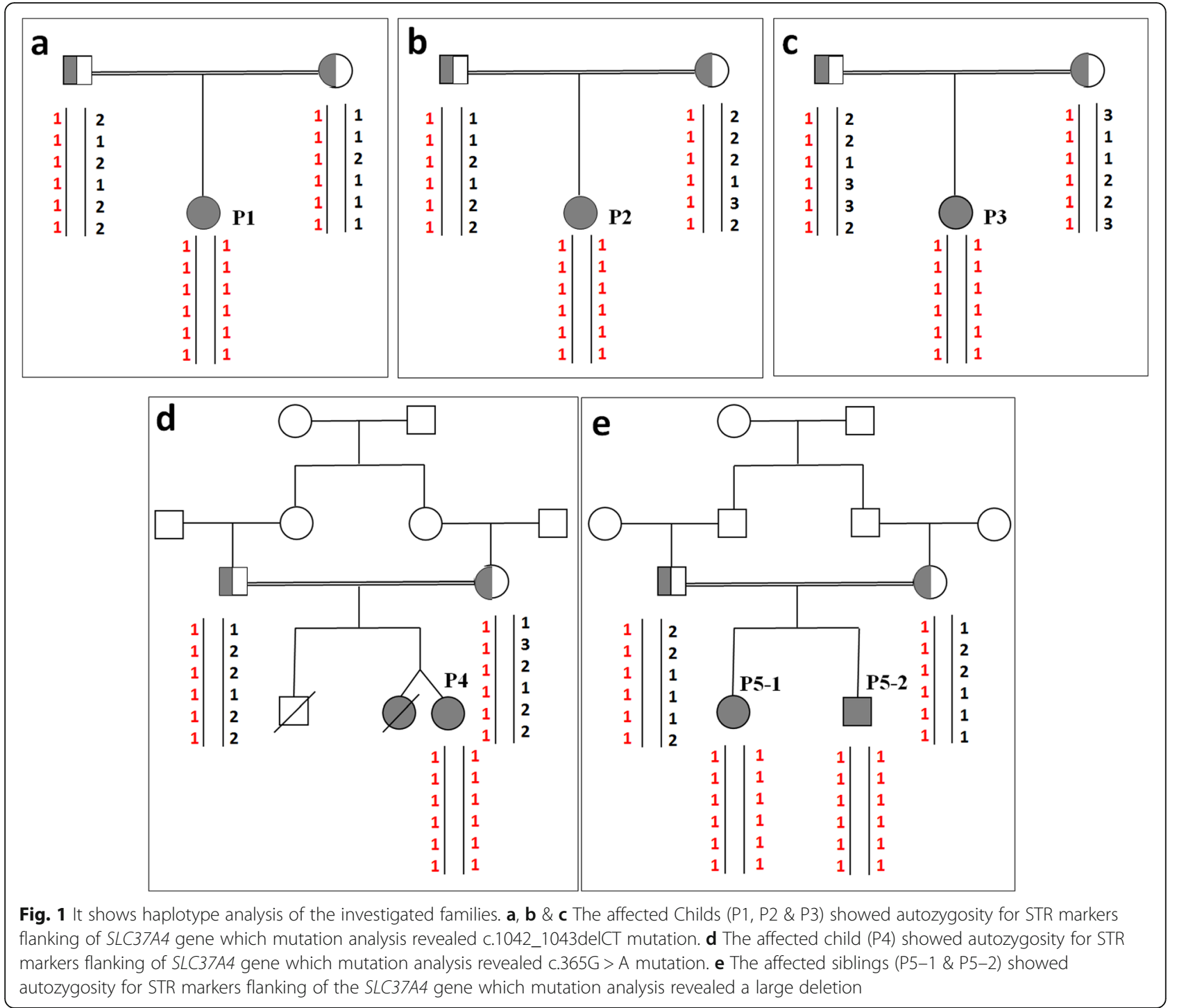

ng/ $\mu$ l DNA. Amplifications of all the exons were carried out under the following program; $95^{\circ} \mathrm{C}$ for $5 \mathrm{~min}, 95^{\circ} \mathrm{C}$ for $30 \mathrm{~s}, 60^{\circ} \mathrm{C}$ for $30 \mathrm{~s}$, and $72^{\circ} \mathrm{C}$ for 40 s repeated by 35 thermal cycles using a thermal cycler (Applied Biosystems, USA) and final step at $72{ }^{\circ} \mathrm{C}$ for $5 \mathrm{~min}$. The novel mutations were named according to the nomenclature recommendations of the Human Genome Variation Society (HGVS) (http://www.hgvs.org).

\section{Long-range PCR assay}

To identify the presence of whole SLC37A4 gene deletion and to confirm the deletion breakpoint sequences, three primers (F1, R1, and R2) were designed using Primer 3 software for two Long-range PCRs and Sanger sequencing. The sequences and chromosome position of these primers were shown in Table 1. In this Long-range PCR assays, the $25 \mu \mathrm{l} \mathrm{PCR} \mathrm{mixture} \mathrm{contains} 12.5 \mu \mathrm{l}$ of

Table 1 The primers characteristics and the size of PCR products used for Long-range PCR assays

\begin{tabular}{lllll}
\hline & Name & Sequence & Chromosome Position (GRCh37) & PCR product size \\
\hline First long-range PCR & F1 & AGCATCACTACTGTTACTCCTCAC & Chr11: 118902469-118902492 & 8276 bp \\
& R1 & GGAGAATGCTGACCCTGATGAC & Chr11: 118894217-118894238 & \\
Second long-range PCR & F1 & AGCATCACTACTGTTACTCCTCAC & Chr11: 118902469-118902492 & 2724 bp \\
& R2 & ATGTCCATGGATCTCAGAGCTTC & Chr11: 118899769-118899791 & \\
\hline
\end{tabular}


LongAmp Taq 2X Master Mix (New England Biolabs), $0.5 \mu \mathrm{l}$ of each $10 \mathrm{pM}$ primer, $10 \mu \mathrm{L} \mathrm{DH} 2 \mathrm{O}$ and $1.5 \mu \mathrm{l}$ of $50 \mathrm{ng} / \mu \mathrm{l}$ DNA. The first Long-range PCR assay was carried out under the following program; $94^{\circ} \mathrm{C}$ for $30 \mathrm{~s}$, $94{ }^{\circ} \mathrm{C}$ for $30 \mathrm{~s}, 62^{\circ} \mathrm{C}$ for $60 \mathrm{~s}$, and $65^{\circ} \mathrm{C}$ for 8 min repeated by 30 amplification cycles and final step at $65^{\circ} \mathrm{C}$ for 10 min. The second Long-range PCR assay was performed with the slightly different program; $94{ }^{\circ} \mathrm{C}$ for 30 s, $94{ }^{\circ} \mathrm{C}$ for $30 \mathrm{~s}, 62^{\circ} \mathrm{C}$ for $50 \mathrm{~s}$, and $65^{\circ} \mathrm{C}$ for 1.5 min repeated by 30 amplification cycles and final step at $65^{\circ} \mathrm{C}$ for $10 \mathrm{~min}$. The PCR products were run using $0.8 \%$ agarose gel electrophoresis.

In silico assessment of pathogenicity of the novel variants Pathogenicity of the variants was investigated using several criteria: (1) population databases such as 1000 genome project (1000 GP) (http://browser.1000genomes. org), dbSNP (http://www.ncbi.nlm.nih.gov/snp), Exome Aggregation Consortium (ExAC) (http://exac.broadinstitute.org), NHLBI GO Exome Sequencing Project (ESP) (http://evs.gs.washington. Edu) were investigated to assess allelic frequency of the variant. (2) in addition, ClinVar (http:// www.ncbi.nlm.nih.gov/clinvar), HGMD (http://www.hgmd.org), Ensemble (https://www.ensembl. org) and recently published articles on PubMed was searched for previously reported variants. (3) A variety of in silico tools consisted of PROVEAN [11], PolyPhen2 [12], MutationTaster [13], HOPE [14], Combined Annotation Dependent Depletion (CADD) [15], and DANN score [16] were used to evaluate the functional effect of the novel variants on the protein. The multiple tools such as PhyloP and PhastCons through UCSC genome browser and GERP were used to investigate the conservation scores [17]. (4) For further confirmation of the pathogenicity of the variants, segregation analysis of the parents was performed using direct sequencing. (5) The identified variants were classified and interpreted according to the ACMG-AMP 2015 Standards and Guidelines [18] that facilitated by the Varsome tool [19].

\section{Results}

\section{Patient phenotypes}

The clinical, biochemical, and hematological parameters of patients with detected causative variants of the SLC37A4 gene are presented in Table 2. There were significant differences in the clinical and biochemical parameters indicating heterogeneity between these GSD1b patients. Consanguinity was detected in all cases. Almost all patients presented hepatomegaly and hypoglycemia. Also, all patients had recurrent infections including otitis, respiratory tract infection, gingivitis, oral candidiasis, pharyngitis, periodic aphthous stomatitis. Hematological findings were different in patients; P1, P2, and P3 with severe neutropenia, $\mathrm{P} 4$ with moderate neutropenia and a sibling (P5-1 and P5-2) with both severe neutropenia and leukopenia (leukocyte count and percentage of neutrophil cells were shown in Table 2). Anemia was observed in all patients except for P4. The other main biochemical parameters were hyperlipidemia, hyperlactatemia, and elevated AST and ALT levels (four patients (66\%)), hypercholesterolemia (two patients (33\%)), hyperuricemia (three patients (66\%)). The clinical information collected from the mentioned patients (P5-1 and P5-2) belongs to the time of disease diagnosis.

$\mathrm{P} 4$ showed hypoglycemia and hepatomegaly in the first year of life. Laboratory examinations showed elevated triglyceride (TG) concentration and uric acid but liver transaminases were normal to slightly increased. Development, growth and facial appearance were normal. Liver biopsy showed severe fatty changes (microvesicular and macrovesicular) and steatosis. The liver was enlarged with normal echogenicity and both kidneys are mildly enlarged. Furthermore, she presented clinically with otitis media, gingivitis, neutropenia (WBC = $7.08^{*} 10^{3} / \mu \mathrm{l}$, Neutrophil count $=966$ ) without leukopenia. Her twin sister had almost similar clinical presentations such as hypoglycemia and hepatomegaly and presented steatosis in liver biopsy. However, she suffered from congenital cataract at the first month of life, growth retardation, epistaxis and elevated creatine phosphokinase (CPK) (data not available) and finally she died due to hypoglycemic coma after 1 year without a definite diagnosis.

Patient P5-1, a 19-year-old girl had suffered from severe neutropenia with leukopenia (WBC $=1.7 * 10^{3} / \mu \mathrm{l}$, Neutrophil count $=289 / \mu \mathrm{l}$ ) that in the first days of life referred to our pediatric clinic with nausea, acidosis, poor feeding, elevated TG $(218 \mathrm{mg} / \mathrm{dl})$, hypoglycemia and hepatomegaly. At sampling date, laboratory examination revealed normal fasting blood glucose, uric acid, TG, and liver transaminases. She had recurrent infectious with otitis, pharyngitis, periodic aphthous stomatitis. Other clinical observations were mild hepatosplenomegaly, enlarged bilateral kidneys, anemia, and increased Erythrocyte Sedimentation Rate (ESR). Chest X-Ray showed bilateral reticular infiltration and decreased bone density. The result of liver biopsy revealed ballooning changes with feathery degeneration and mild steatosis. She had a brother, patient P5-2, a 9-year old boy with similar clinical and laboratory manifestations. $\mathrm{He}$ was brought to the pediatric clinic due to seizure at birth, an elevated level of TG concentration $(464 \mathrm{mg} / \mathrm{dl})$ and moreover, at 4 months, he suffered from severe metabolic acidosis, lethargy, tachypnea, fever, vomiting, poor feeding, and hepatomegaly. Also, he experienced recurrent aphthous stomatitis, neutropenia with leukopenia $\left(\mathrm{WBC}=2.5^{*} 10^{3} / \mu \mathrm{l}\right.$, Neutrophil count $=570$ / $\mu \mathrm{l})$, increased ESR, anemia and severe osteopenia. He 


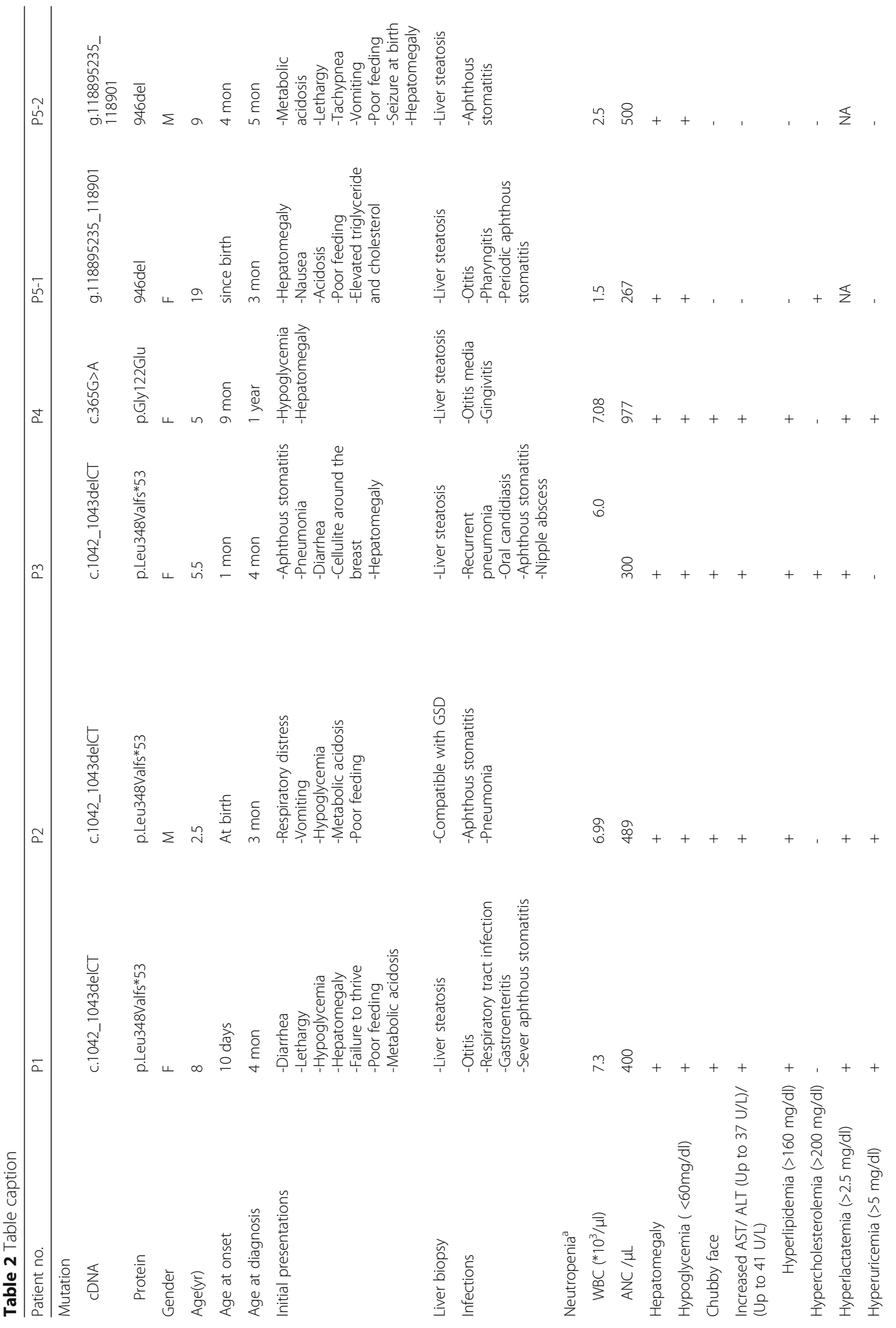




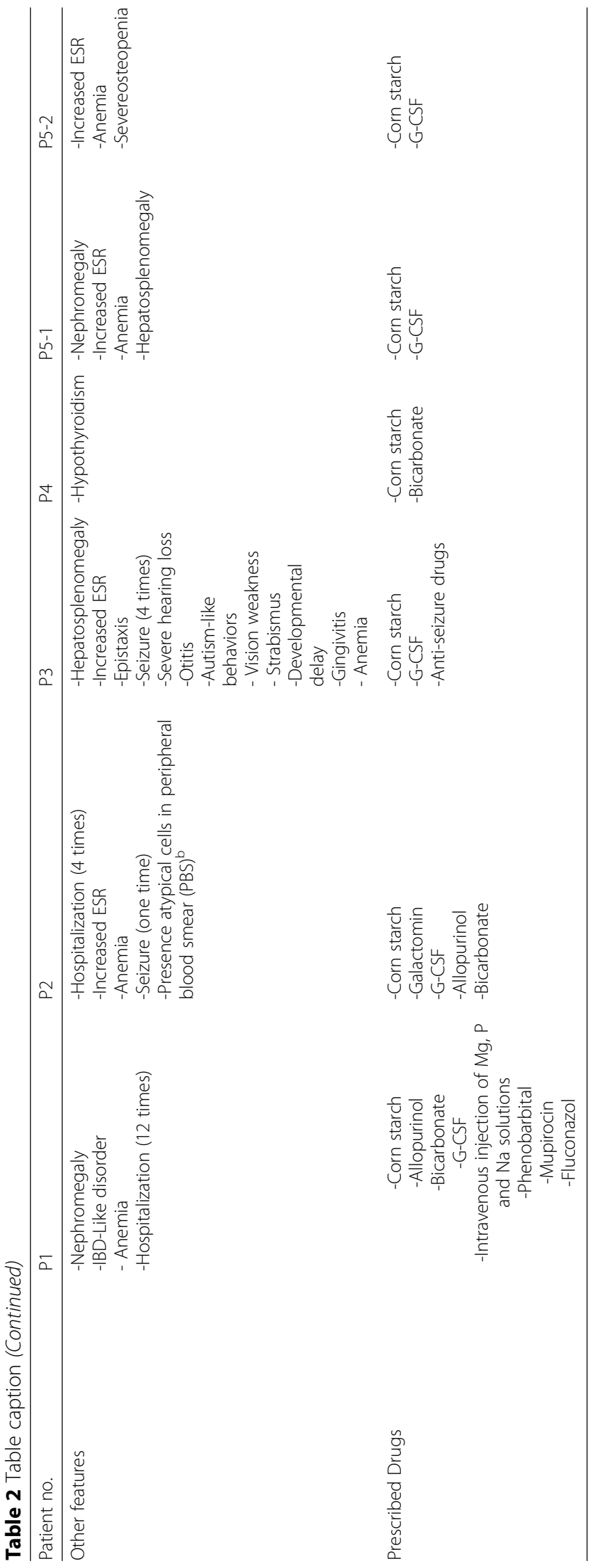


was the second child of the consanguineous family with no family history of GSD.

\section{Molecular analysis of the identified variants}

From the twenty studied patients, six showed autozygous haplotypes for the STR markers flanking the SLC37A4 gene and any patients did not show any autozygosity for the markers flanking the G6PC gene. Sequence analysis of the entire and intron/exon boundaries of the SLC37A4 gene revealed three different mutations in patients with a homozygous haplotype. Haplotype map was shown for families with novel mutations in Fig. 1. Three patients showed two novel mutations (c.365G > A (p.G122E) and whole SLC37A4 gene deletion) and three patients presented a recurrent mutation (c.1042_1043del (p.Leu348Valfs*53)). The recognized mutations in patients were homozygous and were not present in their healthy members of the family. Segregation analysis showed that their parents were heterozygous for the recognized mutations.

\section{The first novel mutation, c.365G > A (p.G122E) in the 4th exon}

One of the two novel variants, c.365G > A (p.G122E) in 4th exon, was identified in the P4 patient that was homozygous for this variant. The twin sister died and her DNA was not available. According to our survey, the variant was not found in any of the population or disease databases as mentioned above. In silico predictive tools showed the deleterious effect on the variant on gene product (Table 3 ). The evolutionary tools showed glycine residue at the position 122 of SLC37A4 protein is highly conserved (PhyloP, PhastCons, and GERP; 4.3 and 1.0 and 5.1, respectively) and multispecies alignment is shown for this variant in UCSC genome browser
(Fig. 2). As predicted by the HOPE project, the mutant residue is bigger and less hydrophobic than the wildtype residue and is negatively charged. According to Varsome tool, this variant is predicted to be variant of uncertain significance (VUS) based on these evidences: (1) The absence of detected variant in any of the population databases (PM2). (2) Missense variant in the SLC37A4 gene that has a low rate of benign missense variation and in which missense variants are a common mechanism of GSD1b disease (PP2) and multiple bioinformatics evidence support a deleterious effect on the gene or the protein (PP3).

\section{The second novel mutation, SLC37A4 gene deletion}

In the sibling (P5-1 and P5-2), a systematic failure to amplify genomic DNA covering all exons of the SLC37A4 gene directed the possible presence of whole gene deletion (g.118895235_118901946del6712 (GRCh37)). To confirm the presence of full-gene deletion and finding the exact breakpoint site, primer walking with the help of three primers (F1, R1, and R2) was done (Table 1). For the first Long-range PCR assay, F1 and R1 primers were designed flanking regions of the suspected deletion (876 bp upstream and 844 bp downstream of the SLC37A4 gene). Sanger sequencing was performed to identify the exact location of the breakpoint site. The results of sequencing established our prediction and identified the location of breakpoint precisely at Chr11:118895235-118,901,946 (Fig. 3). It permitted us to determine the exact size of the deleted region (6712 bp). Gel electrophoresis of this PCR product revealed homozygote deletion in the sibling and heterozygote deletion in parents. The size of DNA fragment in siblings was $1564 \mathrm{bp}$, while the size of the expected genomic segments without deletion was $8276 \mathrm{bp}$ in the control sample, and both segments exist in parents as

Table 3 Table caption

\begin{tabular}{|c|c|c|c|c|}
\hline & Patients & $P 1, P 2$ and $P 3$ & P4 & P5-1 \& P5-2 \\
\hline \multirow[t]{2}{*}{ Variant Definition } & $\begin{array}{l}\text { Varian name } \\
\text { Protein Change }\end{array}$ & $\begin{array}{l}\text { c.1042_1043delCT } \\
\text { p.Leu348Valfs*53 }\end{array}$ & $\begin{array}{l}\text { c.365G }>A \\
\text { G122E }\end{array}$ & g.118895235_118901946del6712 \\
\hline & $\begin{array}{l}\text { Chromosome Position } \\
\text { (GRCh37) } \\
\text { Zygosity }\end{array}$ & $\begin{array}{l}\text { Chr11:118895981_ } \\
118895982 \\
\text { Homozygote }\end{array}$ & $\begin{array}{l}\text { Chr11: } 118898920 \\
\text { Homozygote }\end{array}$ & $\begin{array}{l}\text { Chr11: } 118895235 \text { to chr11: } \\
\text { 118901946 } \\
\text { Homozygote }\end{array}$ \\
\hline In-silico Predictive Tools & $\begin{array}{l}\text { CADD (Phred Score) } \\
\text { DANN } \\
\text { Polyphen-2 } \\
\text { PROVEAN }\end{array}$ & $\begin{array}{l}\text { 35(Deleterious) } \\
- \\
- \\
-\end{array}$ & $\begin{array}{l}27.4 \text { (Deleterious) } \\
0.82 \text { (Deleterious) } \\
0.958 \\
\text { (PROBABLYDAMAGING) } \\
2.66 \text { (Deleterious) }\end{array}$ & $\begin{array}{l}- \\
- \\
- \\
-\end{array}$ \\
\hline $\begin{array}{l}\text { Population/Disease } \\
\text { Databases }\end{array}$ & $\begin{array}{l}1000 \text { GP } \\
\text { EXAC } \\
\text { GenomAD } \\
\text { ESP } \\
\text { HGMD } \\
\text { Clinvar }\end{array}$ & $\begin{array}{l}- \\
0.00023 \\
0.00018 \\
0.0003 \\
\text { CD982664 } \\
\text { VCV000006926 }\end{array}$ & $\begin{array}{l}- \\
- \\
- \\
- \\
- \\
-\end{array}$ & $\begin{array}{l}- \\
- \\
- \\
- \\
- \\
-\end{array}$ \\
\hline
\end{tabular}




\begin{tabular}{|c|c|c|c|c|c|c|c|c|c|c|c|c|c|c|c|c|c|c|c|c|c|c|}
\hline Human & K & $\mathbf{R}$ & L & v & $\mathrm{K}$ & G & C & $\mathbf{P}$ & P & W & G & L & G & Q & A & L & G & $\mathrm{N}$ & L & $F$ & W & L \\
\hline Chimp & K & $\mathbf{R}$ & L & v & K & G & c & $\mathbf{P}$ & P & w & G & L & G & Q & A & L & G & N & L & $\mathbf{F}$ & w & L \\
\hline Gorilla & K & $\mathbf{R}$ & L & v & $\mathrm{K}$ & G & C & $\mathbf{P}$ & $\mathbf{P}$ & w & G & L & G & $Q$ & A & L & G & N & L & $\mathrm{F}$ & w & L \\
\hline Gibbon & K & $\mathbf{R}$ & L & v & $\mathrm{K}$ & G & C & P & P & w & G & L & G & $Q$ & A & L & G & N & L & $\mathrm{F}$ & w & L \\
\hline Rhesus & K & $\mathbf{R}$ & L & v & $\mathrm{K}$ & G & c & $\mathbf{P}$ & P & w & G & L & G & Q & A & L & G & $\mathrm{N}$ & L & $\mathrm{F}$ & w & L \\
\hline Marmoset & K & $\mathbf{R}$ & L & v & K & G & c & $\mathbf{P}$ & P & w & G & L & G & Q & A & L & G & N & L & $F$ & w & L \\
\hline Chinese_hamster & $\mathrm{K}$ & $\mathbf{R}$ & L & I & $\mathrm{K}$ & G & c & $\mathbf{P}$ & $\mathbf{P}$ & w & G & L & G & Q & A & L & G & $\mathrm{N}$ & L & $\mathrm{F}$ & w & L \\
\hline Golden_hamster & $\mathrm{K}$ & $\mathbf{R}$ & $\mathrm{L}$ & I & $\mathrm{K}$ & G & c & $\mathbf{P}$ & $\mathbf{P}$ & w & G & L & G & Q & A & L & G & N & L & $\mathrm{F}$ & w & L \\
\hline Mouse & $\mathrm{k}$ & $\mathbf{R}$ & $\mathrm{L}$ & I & $\mathrm{K}$ & G & c & $\mathbf{P}$ & $\mathbf{P}$ & w & G & L & G & Q & A & L & G & $\mathrm{N}$ & L & $\mathrm{F}$ & w & L \\
\hline Rat & K & $\mathbf{R}$ & L & I & $\mathrm{K}$ & G & c & $\mathbf{P}$ & $\mathbf{P}$ & w & G & $\mathrm{L}$ & G & Q & A & L & G & $\mathrm{N}$ & L & $\mathbf{F}$ & w & L \\
\hline Rabbit & $\mathrm{K}$ & $\mathbf{R}$ & L & v & $\mathrm{K}$ & G & c & $\mathbf{P}$ & P & w & G & L & G & Q & A & L & G & N & L & $\mathbf{F}$ & w & L \\
\hline Pika & K & $\mathbf{R}$ & L & I & K & G & C & $\mathbf{P}$ & P & W & G & L & G & Q & A & L & G & N & L & $F$ & W & L \\
\hline Pig & $\mathrm{K}$ & $\mathbf{R}$ & L & I & $\mathrm{K}$ & G & c & $\mathbf{P}$ & $\mathbf{P}$ & w & G & $\mathrm{L}$ & G & Q & A & L & G & $\mathrm{N}$ & L & $\mathbf{F}$ & w & L \\
\hline Dolphin & K & $\mathbf{R}$ & L & v & $\mathrm{K}$ & G & c & $\mathbf{P}$ & P & w & G & L & G & Q & A & L & G & N & L & $\mathrm{F}$ & w & L \\
\hline Cow & K & $\mathbf{R}$ & L & v & K & G & C & $\mathbf{P}$ & P & w & G & L & G & Q & A & L & G & N & L & $\mathrm{F}$ & w & L \\
\hline Sheep & $\mathrm{K}$ & $\mathbf{R}$ & L & v & $\mathrm{K}$ & G & c & $\mathbf{P}$ & P & w & G & L & G & Q & A & L & G & $\mathrm{N}$ & L & $\mathrm{F}$ & W & L \\
\hline Horse & K & $\mathbf{R}$ & L & v & K & G & c & A-C & P & w & G & L & G & Q & A & L & G & N & L & F & w & L \\
\hline Cat & K & $\mathbf{R}$ & L & I & $\mathrm{K}$ & G & C & $\mathbf{P}$ & $\mathbf{P}$ & w & G & L & G & Q & A & L & G & N & L & $\mathbf{F}$ & w & L \\
\hline Dog & K & $\mathbf{R}$ & L & I & K & G & c & P & P & w & G & L & G & Q & A & L & G & N & L & $\mathrm{F}$ & w & L \\
\hline Hedgehog & $\mathrm{K}$ & $\mathbf{R}$ & L & I & K & G & c & $\mathbf{P}$ & P & w & G & L & G & $Q$ & A & L & G & N & L & $\mathrm{F}$ & w & L \\
\hline Elephant & K & $\mathbf{R}$ & L & I & K & G & C & $\mathbf{P}$ & P & w & G & L & G & Q & A & L & G & N & L & $F$ & w & L \\
\hline Chicken & K & $\mathbf{R}$ & L & v & K & G & C & $\mathbf{P}$ & P & w & G & L & G & Q & A & L & G & N & L & F & w & L \\
\hline X_tropicalis & $\mathrm{K}$ & $\mathbf{R}$ & L & v & K & G & c & $\mathbf{P}$ & P & w & G & $\mathrm{L}$ & G & Q & A & L & G & $\mathbf{N}$ & $\mathrm{F}$ & $\mathrm{F}$ & w & L \\
\hline Medaka & $\mathrm{K}$ & $\mathbf{R}$ & L & I & $\mathrm{R}$ & G & c & $\mathbf{P}$ & $\mathbf{P}$ & w & G & L & G & $Q$ & G & L & G & N & $\mathrm{F}$ & $\mathrm{F}$ & w & L \\
\hline
\end{tabular}

Fig. 2 Multispecies alignment for the identified variant in P4 patient: c.365G > A, p.G122E. The panel from the UCSC genome browser (https://genome.ucsc.edu/cgi-bin/)

expected. To further confirm the presence of deletion, the second Long-range PCR assay with another set of primers (F1 and R2) was used to discriminate between cases with and without deletion. R2 primer was designed around the 4th exon as an internal control. To visually confirm the mutant and wide type alleles, PCR products were run on gel electrophoresis and generated a 2724 bp fragment in parents. There was no amplification in patients bearing the deletion.

\section{Discussion}

GSD1 is the most prevalent hepatic type of Glycogen storage diseases (GSDs) which comprises a group of autosomal recessive disorders characterized by the deficiency of the enzymes that regulate the synthesis or degradation of glycogen. GSD1 categorized into two overlapping forms, $1 \mathrm{a}$ and $1 \mathrm{~b}$ which caused by the deficiency of G6PC and SLC37A4 genes, respectively [1]. The high rate of consanguineous marriage in Iran proposes a high incidence of autosomal recessive disorders. Since GSD1 is a rather rare disease in Iran and other populations, only a few studies have been published regarding the GSD1 mutational spectrum in Iran and elsewhere [21], so we decided to conduct autozygosity mapping to quick and indirectly find the mutated gene in nineteen families suspected to GSD. Haplotype analysis of the studied families showed six patients with autozygous haplotype block for the SLC37A4 gene and no family was autozygous for the markers flanking the G6PC gene. Subsequently, the sequencing of the SLC37A4 gene was revealed two novel and one recurrent mutation in six patients. This is the first study to summarize the clinical and molecular characteristics of Iranian patients with GSD Ib.
SLC37A4 deficiencies affect metabolic and myeloid phenotypes. In metabolic phenotype, in gluconeogenic organs of liver, kidney, and intestine, SLC37A4 and G6PC together are required to maintain interprandial blood glucose homeostasis. In myeloid phenotype, SLC37A4 and G6PC3 together are required to maintain neutrophil homeostasis and their deficiency leads to immune deficiency, characterized by neutropenia and neutrophil dysfunction [3]. Previous Studies have shown that GSD-Ib patients exhibit an increased risk of developing autoimmune disorders, including IBD, thyroid autoimmunity, and myasthenia gravis. Melis and colleagues exhibited that GSD-Ib patients indicated lymphopenia and T cells exhibit altered glycolysis and impaired peripheral regulatory $\mathrm{T}$ cell function [22]. In previous studies, the most common mutation was c.1042_1043del (p.Leu348Valfs*53) that has been recurrently reported in German (32\%) populations and mixed Caucasian (27-31\%) [23]. The mentioned deletion in the 8th exon leads to the deficiency of enzyme activity which accompanied by severe neutropenia in three patients in our study (P1, P2, and P3). These three patients have several hospitalizations due to hypoglycemia and seizure attacks. In addition to GSD1b common symptoms (OMIM:232220), P3 suffered from secondary symptoms such as vision weakness, severe hearing loss, strabismus, developmental delay and autism-like behaviors that had not been reported with this disease in previous publications. These signs may be due to recurrent severe seizures.

Regarding the novel c.365G > A (p.G122E) mutation in $\mathrm{P} 4$ patient, glycine at the transmembrane domain of the glucose 6-phosphate translocase enzyme is more hydrophobic than the mutant residue. This difference in hydrophobicity can affect the hydrophobic interactions with the membrane lipids. Furthermore, glycine is the most flexible of all residues. This flexibility might be necessary for the 

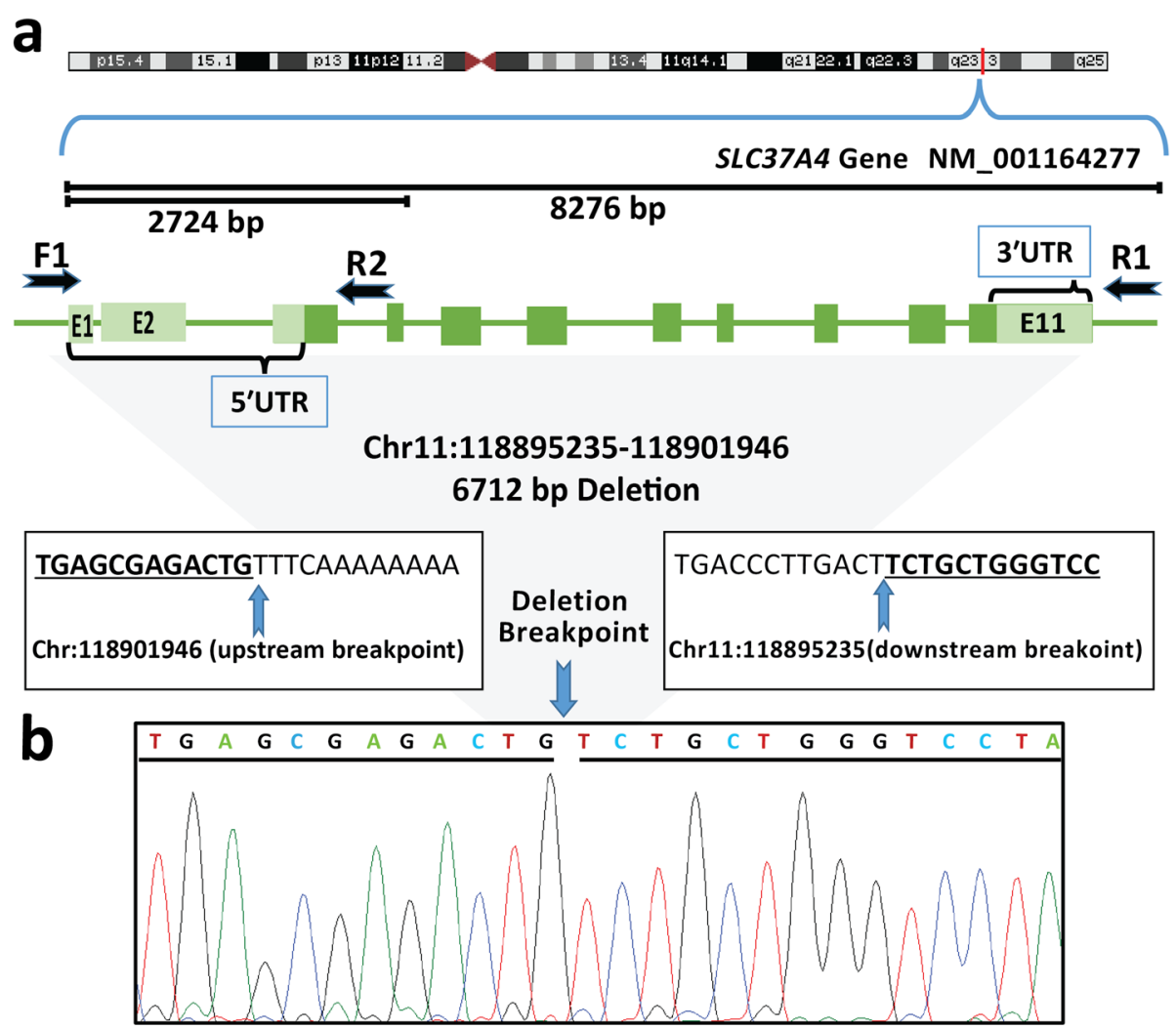

C

P5-1 P5-2 F M CT
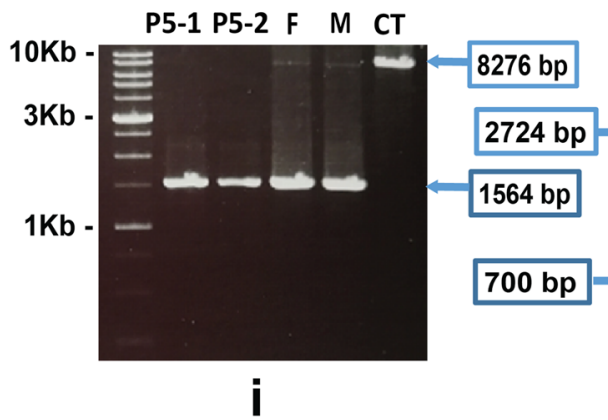

P5-1 P5-1 F M CT ntc

$10 \mathrm{~Kb}$

P5-1 P5-1 F M CT ntc ladder

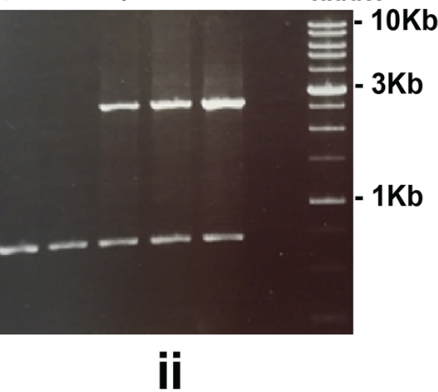

Fig. 3 Long-range PCR and sequencing showed the full-gene deletion of SLC37A4 in the siblings (P5-1 and P5-2) with GSD1b. a the gene transcript image (was taken from Genome Data Viewer in NCBI) and the orientation of the designed primers across upstream and downstream breakpoint. The black arrows indicate the position of primers used in Long-range PCR. The length of the genomic segment for each set of primers (F1\&R1 and F1\&R2) was shown. b Sanger sequencing result of the breakpoint site and flanking region. Two boxes above the sequencing result indicate the sequences across upstream and downstream breakpoint. In Sanger sequencing result, the blue arrow shows the breakpoint and a 6712 bp sequence deletion on chr11 of the human reference genome (GRCh37). c Gel electrophoresis of the PCR product. i) Results of the first long-range PCR (PCR with F1 and R1 primers) are presented in the left which shows this segment in the siblings, parents and control samples. Lane 1 contains a $10 \mathrm{~kb}$ ladder, lane 2 and 3 contains the products of deleted allele, lane 3 and 4 contains the products of both the deleted allele and the wide type. Lane 5 contains the wide-type allele. ii) Results of the second long-range PCR (PCR with F1 and R2) are presented in the right which shows this segment in two siblings, parents and control samples. Lane 1 contains a $10 \mathrm{~kb}$ ladder, lane 2 contains NTC, Lane 3, 4 and 5 contains a 2724 bp fragment without deletion and lane 6 and 7 contains no amplification. All lanes (except lane 2) include a 700 bp internal control (Exon 5 of G6PC gene). The products of deleted allele, (1564 bp); the products of wide-type allele, (8276 bp); M, mother; F, father; CT, control sample

protein's function [14]. Mutation of this glycine can abolish this function and coincidently, the torsion angles of this residue are incorrect. In silico analysis tools were consistent in predicting that this variant may impair protein function and this substitution can change the enzyme conformation. The hematological findings in this patient showed moderate neutropenia in contrast to P1, P2, and P3 patients (frameshift variant) with severe neutropenia. 
The CADD score, which has high sensitivity to predict molecular pathogenicity of variants, was 27 and 35 in missense variant (p.G122E) and frameshift variant (p.Leu348Valfs"53), respectively. Since the higher values of CADD score predict more severe effect, it may explain the pronounced hematological finding in P1, P2, and P3 patients in comparison to $\mathrm{P} 4$.

The second novel mutation, whole SLC37A4 gene deletion, was identified in a sibling (P5-1 and P5-2). The homozygote $6.7 \mathrm{~kb}$ deletion which spans near the entire SLC37A4 gene can lead to the complete loss of function of both alleles. Therefore, G6PT protein will not be produced. To date, 116 mutations were identified for SLC37A4 gene (http://www.hgmd.org) including 86 substitutions and $30 \mathrm{small} / \mathrm{gross}$ deletion and insertions. To the best of our knowledge, this is the first report of whole SLC37A4 gene deletion. Here, the siblings (P5-1 and P5-2) had anemia, leucopenia, and severe neutropenia with increased lymphocyte counts. These hematological findings suggest a correlation between entire SLC37A4 gene deletion and severe myeloid problems in GSD-Ib.

However, in previous studies, no correlation was reported between individual mutations and present/absence of neutropenia, bacterial infections, and other complications [24-26]. Recently, one study by Sarajlija A et al., revealed some specific mutations in SLC37A4 have an impact on the severity of neutropenia and the capacity of increasing neutrophil count in serious bacterial infections (SBI) [27]. It is worth to mention that all GSD1b patients did not have neutropenia and some of them may suffer from cyclic neutropenia [5]. This phenotypic variability of GSD $1 \mathrm{~b}$ might suggest contribution of one or several other factors (ie., as modifier genes) in phenotype disease, which could change "simple" Mendelian disorders into complex traits [28].

\section{Conclusions}

Clinical and biochemical parameters were different in the GSD1b patients. Therefore, the strict genotypephenotype correlation couldn't be demonstrated based on this data. However, hematological findings revealed a correlation between causative mutations and myeloid phenotypes. P1, P2, and P3 with frameshift variant (p.Leu348Valfs"53) had severe neutropenia. P4 with a missense mutation (p.G122E) showed moderate neutropenia. The sibling (P5-1 and P5-2) with whole SLC37A4 deletion represented both severe neutropenia and leukopenia. This large deletion was accompanied by severe impairment of myeloid cells. But establishing a definitive genotype-phenotype correlation would needs more studies with larger sample sizes. Since GSD is a group of clinically and genetically heterogeneous disorders, it is recommended to use whole-exome sequencing
(WES) for the detection of causative mutations in families with no autozygous haplotype block for the markers flanking the $G 6 P C$ gene.

\begin{abstract}
Abbreviations
1000 GP: 1000 genome project; ALT: Alanine transaminase; AST: Aspartate aminotransferase; CADD: Combined Annotation Dependent Depletion; CPK: Creatine phosphokinase; ESP: Exome Sequencing Project;

ESR: Erythrocyte Sedimentation Rate; ExAC: Exome Aggregation Consortium; G6Pase-a: Glucose-6-phosphatase-a; G6PT: Glucose-6-phosphate transporter; GSD: Glycogen storage disease; HGVS: Human Genome Variation Society; IBD: Inflammatory bowel disease; SBI: Serious bacterial infections;

SERV: Sequence-based Estimation of Repeat Variability; STR: Short tandem repeat; TG: Triglyceride; TRF: Tandem Repeat Finder; VUS: Variant of uncertain significance; WBC: White blood cell; WES: Whole-exome sequencing
\end{abstract}

\section{Acknowledgments}

The current study was supported by a Grant from the Tehran University of Medical Sciences. The authors extend their appreciation to the patients and their preparedness to participate in this study.

\section{Authors' contributions}

EM performed experiments and analysis and interpretation of the data and drafting the manuscript. AM was involved in drafting the manuscript. TS was involved in patients counseling and in-silico analysis of datasets. NZ was involved in molecular testing. SM, RP, AH, and NM contributed to patients' assessments. YF was involved in drafting the manuscript. RA contributed in patients' assessments and data collection. $\mathrm{MMH}$ designed and supervised the study and in drafting and finalizing the manuscript. All authors have read and approved the final manuscript.

\section{Funding}

The project was supported by a grant from Tehran University of Medical Sciences.

\section{Availability of data and materials}

The datasets are available from the corresponding author on reasonable request.

Ethics approval and consent to participate

The study protocol was approved by the ethical committee of Tehran University of Medical Sciences. The study consent forms have been attained from the participants and all participants signed informed consent forms.

Consent for publication

The consent to publish has been obtained from the participant to report individual patient data.

\section{Competing interests}

The authors declare that they have no competing interests.

\section{Author details}

${ }^{1}$ Department of Medical Genetics, Faculty of Medicine, Tehran University of Medical Sciences, Tehran, Iran. ${ }^{2}$ Department of Medical Genetics and Molecular Biology, Faculty of Medicine, Iran University of Medical Sciences, Tehran, Iran. ${ }^{3}$ Department of Molecular Medicine, School of Advanced Technologies in Medicine, Tehran University of Medical Sciences, Tehran, Iran. ${ }^{4}$ Department of Pediatric Endocrinology and Metabolism, Mofid Children's Hospital, Shahid Beheshti University of medical sciences, Tehran, Iran. ${ }^{5}$ Growth and Development Research Center, Department of

Endocrinology, Children's Medical Center, Tehran University of Medical

Sciences, Tehran, Iran. ${ }^{6}$ Department of Gastroenterology, Children's Medical Center, Tehran University of Medical Science, Tehran, Iran. 
Received: 5 August 2019 Accepted: 2 December 2019

Published online: 31 January 2020

\section{References}

1. Chou J, Matern D, Mansfield BC, Chen Y-T. Type I glycogen storage diseases: disorders of the glucose-6-phosphatase complex. Curr Mol Med. 2002;2(2): 121-43.

2. Chou JY, Jun HS, Mansfield BC. Glycogen storage disease type I and G6Pase- $\beta$ deficiency: etiology and therapy. Nat Rev Endocrinol. 2010;6(12):676.

3. Chou JY, Jun HS, Mansfield BC. Type I glycogen storage diseases: disorders of the glucose-6-phosphatase/glucose-6-phosphate transporter complexes. J Inherit Metab Dis. 2015;38(3):511-9.

4. Visser G, Rake J-P, Fernandes J, Labrune P, Leonard JV, Moses S, et al. Neutropenia, neutrophil dysfunction, and inflammatory bowel disease in glycogen storage disease type lb: results of the European study on glycogen storage disease type I. J Pediatr. 2000;137(2):187-91.

5. Kure S, Hou D-C, Suzuki Y, Yamagishi A, Hiratsuka M, Fukuda T, et al. Glycogen storage disease type Ib without neutropenia. J Pediatr. 2000; 137(2):253-6.

6. Davit-Spraul A, Piraud M, Dobbelaere D, Valayannopoulos V, Labrune P, Habes D, et al. Liver glycogen storage diseases due to phosphorylase system deficiencies: diagnosis thanks to non invasive blood enzymatic and molecular studies. Mol Genet Metab. 2011;104(1-2):137-43.

7. Saadat $\mathrm{M}$, Ansari-Lari M, Farhud D. Short report consanguineous marriage in Iran. Ann Hum Biol. 2004;31(2):263-9.

8. Miller $\mathrm{S}$, Dykes $\mathrm{D}$, Polesky $\mathrm{H}$. A simple salting out procedure for extracting DNA from human nucleated cells. Nucleic Acids Res. 1988;16(3):1215.

9. Benson G. Tandem repeats finder: a program to analyze DNA sequences. Nucleic Acids Res. 1999;27(2):573-80.

10. Legendre M, Pochet N, Pak T, Verstrepen KJ. Sequence-based estimation of minisatellite and microsatellite repeat variability. Genome Res. 2007;17(12): 1787-96.

11. Choi Y, Chan AP. PROVEAN web server: a tool to predict the functional effect of amino acid substitutions and indels. Bioinformatics. 2015;31(16): 2745-7.

12. Adzhubei I, Jordan DM, Sunyaev SR. Predicting functional effect of human missense mutations using PolyPhen-2. Curr Protoc Hum Genet. 2013;76(1): $1-741$.

13. Schwarz JM, Cooper DN, Schuelke M, Seelow D. MutationTaster2: mutation prediction for the deep-sequencing age. Nat Methods. 2014;11(4):361.

14. Venselaar $H$, te Beek TA, Kuipers RK, Hekkelman ML, Vriend G. Protein structure analysis of mutations causing inheritable diseases. An e-Science approach with life scientist friendly interfaces. BMC Bioinformatics. 2010; 11(1):548.

15. Kircher M, Witten DM, Jain P, O'Roak BJ, Cooper GM, Shendure J. A general framework for estimating the relative pathogenicity of human genetic variants. Nat Genet. 2014;46(3):310.

16. Quang D, Chen Y, Xie X. DANN: a deep learning approach for annotating the pathogenicity of genetic variants. Bioinformatics. 2014;31(5):761-3.

17. Davydov EV, Goode DL, Sirota M, Cooper GM, Sidow A, Batzoglou S. Identifying a high fraction of the human genome to be under selective constraint using GERP++. PLoS Comput Biol. 2010;6(12):e1001025.

18. Richards S, Aziz N, Bale S, Bick D, Das S, Gastier-Foster J, et al. Standards and guidelines for the interpretation of sequence variants: a joint consensus recommendation of the American College of Medical Genetics and Genomics and the Association for Molecular Pathology. Genet Med. 2015;17(5):405.

19. Kopanos C, Tsiolkas V, Kouris A, Chapple CE, Aguilera MA, Meyer R, et al. VarSome: the human genomic variant search engine. Bioinformatics. 2019:35(11):1978.

20. Al-Gwaiz LA, Babay HH. The diagnostic value of absolute neutrophil count, band count and morphologic changes of neutrophils in predicting bacterial infections. Med Princ Pract. 2007;16(5):344-7.

21. Mahmoud SK, Khorrami A, Rafeey M, Ghergherehchi R, Sima MD. Molecular analysis of glycogen storage disease type la in Iranian Azeri Turks: identification of a novel mutation. J Genet. 2017;96(1):19-23.

22. Melis D, Carbone F, Minopoli G, La Rocca C, Perna F, De Rosa V, et al. Cutting edge: increased autoimmunity risk in glycogen storage disease type $1 \mathrm{~b}$ is associated with a reduced engagement of glycolysis in T cells and an impaired regulatory T cell function. J Immunol. 2017;198(10):3803-8.
23. Kishnani PS, Austin SL, Abdenur JE, Arn P, Bali DS, Boney A, et al. Diagnosis and management of glycogen storage disease type l: a practice guideline of the American College of Medical Genetics and Genomics. Genet Med. 2014;16(11):e1.

24. Melis D, Fulceri R, Parenti G, Marcolongo P, Gatti R, Parini R, et al. Genotype/ phenotype correlation in glycogen storage disease type $1 \mathrm{~b}$ : a multicentre study and review of the literature. Eur J Pediatr. 2005;164(8):501-8.

25. Martens DH, Kuijpers TW, Maianski NA, et al. A patient with common glycogen storage disease type lb mutations without neutropenia or neutrophil dysfunction. J Inherit Metab Dis. 2006;29:224-5.

26. Angaroni $\mathrm{CJ}$, Labrune $\mathrm{P}$, Petit $\mathrm{F}$, et al. Glycogen storage disease type Ib without neutropenia generated by a novel splice-site mutation in the glucose-6-phosphate translocase gene. Mol Genet Metab. 2006;88(1):96-9. Epub 2006 Feb 21

27. Sarajlija A, Djordjevic M, Kecman B, Skakic A, Pavlovic S, Pasic S, et al. Impact of genotype on neutropenia in a large cohort of Serbian patients with glycogen storage disease type Ib. Eur J Med Genet. 2019:103767. https://doi. org/10.1016/j.jmg.2019.103767.

28. Dipple KM, McCabe ER. Phenotypes of patients with "simple" Mendelian disorders are complex traits: thresholds, modifiers, and systems dynamics. Am J Hum Genet. 2000;66(6):1729-35.

\section{Publisher's Note}

Springer Nature remains neutral with regard to jurisdictional claims in published maps and institutional affiliations.

\section{Ready to submit your research? Choose BMC and benefit from:}

- fast, convenient online submission

- thorough peer review by experienced researchers in your field

- rapid publication on acceptance

- support for research data, including large and complex data types

- gold Open Access which fosters wider collaboration and increased citations

- maximum visibility for your research: over $100 \mathrm{M}$ website views per year

At BMC, research is always in progress.

Learn more biomedcentral.com/submissions 